\title{
Hand Assisted Thoracoscopy: Reviving a Novel Technique
}

\author{
M Kaushik ${ }^{1 *}$, J Kurle ${ }^{2}$ and B Mensink ${ }^{2}$ \\ ${ }^{1}$ Dept of Surgery Ascension Providence Hospital, Michigan State University, USA \\ 2Dept of Surgery, Sinai Grace Hospital, USA
}

\section{Editorial \\ Volume 3 Issue 1}

Received Date: September 16, 2019

Published Date: September 18, 2019

*Corresponding author: Manu Kaushik MD, Department of General Surgery, Ascension Providence Hospital, Michigan

State University, Southfield MI, USA, Email: manukaushik88@gmail.com

\section{Editorial}

Video Assisted Thoracoscopic Surgery (VATS) has revolutionalized the field of thoracic surgery and has served an important role in planning the approach to surgical management of pulmonary and cardiac pathology in the recent decades. Jacobeaus from Sweden performed one of the initial thoracoscopies under local anesthesia to evaluate and treat pleural effusions in a patient with pulmonary tuberculosis [1]. The technique and methods have since evolved, and like other minimally invasive procedures have often replaced their open counterparts in most centers. VATS is known to have shorter operative times, less intraoperative bleeding and shorter hospital stay.

Hand Assisted Thoracoscopic surgery (HATS) is a novel minimally invasive approach for performing techniques conventionally performed by posterolateral thoracotomy. This technique can act as a bridge between VATS and open thoracotomy and can be used in selective cases where there appears a need to convert to open secondary to difficult anatomy, inability to perform one lung ventilation etc. This technique can reduce the number of open thoracotomies and reduce hospital stay, improve quality of life and faster rehabilitation. There have been multiple reports of HATS for pulmonary metastectomy through the sternocostal triangle allowing access for manual palpation of both lungs [2].

VATS was introduced as a minimally invasive surgery and was quickly utilized in metastectomy. Approaches like trans-xiphoid HATS developed by Mineo and Detterbeck as well as trans-diaphragmatic HATS developed by Wight [2]. Although these procedures have revolutionized thoracoscopic surgery by allowing it's use to treat a wider range of pathologies, they do not provide the solution for patients who are unable to undergo single lung ventilation often resulting in conversion to open thoracotomy for exposure.

At our institution, we have been utilizing a new method of hand assisted thoracoscopic surgery. During cases in which single lung ventilation is not possible or in which there is poor visualization with the camera due to anatomic or pathologic abnormalities, we extend the anterolateral VATS incision to $8-10 \mathrm{~cm}$ for use as a direct intrathoracic hand port. This is much akin to the use of a hand port in minimally invasive surgery of the abdomen. By using a hand port in this fashion, we have been able to successfully and safely provide retraction and assistance with camera guidance to prevent conversion to open thoracotomy in patients unable to tolerate single lung ventilation. A small malleable retractor can also be introduced through a different trochar incision to aid in countertraction if necessary. This so called Kurle-Kaushik Maneuver has been used at our institution as a method of hand assisted thoracoscopic surgery to successfully treat retained hemothorax and empyema in polytrauma patients unable to undergo single lung ventilation and has prevented the need for conversion to open thoracotomy in these patients.

VATS has been widely used in the past 2 decades. Although it's minimally invasive nature is of great benefit, its application is somewhat limited compased to laparoscopic surgery due to the inability insufflate the thoracic cavity to allow proper room for exposure. Additionally, palpation of the lungs can be difficult and incomprehensive and therefore traditional VATS may not be suitable for procedures like pulmonary metastasectomy [2]. While the latter problem can be addressed with trans-xiphoid and trans-diaphragmatic hand ports, we have found the Kurle-Kaushik Maneuver 


\section{International Journal of Surgery \& Surgical Techniques}

to be a less invasive method of preventing conversion to open thoracotomy during simple thoracoscopic procedures in which anatomy or lung hyperinflation prevents safe and adequate exposure.

\section{References}

1. Wright GM, Clarke CP, Paiva JM. Hand Assisted thoracoscopic surgery. Ann Thoracic Surg 2003 May; 75(5):1665-7
2. Hao L, Long J, Yong Bin L et al. Hand-assisted thoracoscopic surgery for pulmonary metastasectomy through sternocostal triangle access: superiority in detection of non imaged pulmonary nodules. Sci Rep. 2014 Apr 1;4:4539 